\title{
GENERALIZED RAMSEY THEORY VI: RAMSEY NUMBERS FOR SMALL PLEXES
}

\author{
RICHARD A. DUKE and FRANK HARARY*
}

(Received 18 March 1975; revised 15 July 1975)

Communicated by W. D. Wallis

\begin{abstract}
Generalized Ramsey theory for graphs was formulated and developed in the previous papers in this series. We extend the area here by introducing generalized Ramsey numbers for higher dimensional simplicial complexes. In particular we calculate explicitly the Ramsey numbers for several small "pure 2-complexes", or more briefly plexes, in which each edge is contained in some 2-cell.
\end{abstract}

\section{Introduction}

The classical Ramsey theory for graphs involves the occurrence of monochromatic complete subgraphs in line-colored complete graphs. It follows from the result known as Ramsey's theorem, first proved in Ramsey (1930), that for any positive integers $m$ and $n$, there exists an integer $p$ such that in every coloring of the lines of $K_{p}$, the complete graph on $p$ points, with two colors, green and red, either the green subgraph contains a copy of $K_{m}$ or the red subgraph contains a copy of $K_{n}$. The Ramsey number $r(m, n)$ is the smallest value of $p$ for which this is so. Despite a great deal of research, only a few non-trivial Ramsey numbers are known [see Harary (1969), p. 17 or Graver and Yackel (1968)].

Recently considerable attention has been devoted to a generalization of the classical Ramsey theory. In this generalized theory, for each pair of graphs $G$ and $H$, the number $r(G, H)$ denotes the least integer $p$ such that in every coloring of the lines of $K_{p}$ with the colors green and red, the green subgraph contains a copy of $G$ or the red subgraph contains a copy of $H$. The existence of

* Research supported in part by the Air Force Office of Scientific Research. 
these numbers for each $G$ and $H$ follows from the classical result. For surveys of the large quantity of information that has already been collected on this topic see Burr (1974) and Harary (1974).

A more general form of the original Ramsey theorem [see Ryser (1963), p. 39] implies that for any positive integers $k, m$, and $n$ and any coloring with green and red of the $k$-sets (that is, $k$-element subsets) of a sufficiently large set $A$, there exists either an $m$-set of $A$ each of whose $k$-sets is colored green or an $n$-set of $A$ each of whose $k$-sets is colored red. For $k=2$ this is the graph-theoretic result described above. In general the statement can be reformulated in the language of finite simplicial complexes, and we shall be interested here in the case where $\boldsymbol{k}=\mathbf{3}$ for which we have the following version: given any positive integers $m$ and $n$, and any coloring with green and red of the 2-cells (triples) of a complete 2-complex with sufficiently many vertices, there exists either a complete 2-complex on $m$ vertices each of whose 2-cells is green or a complete 2-complex on $n$ vertices each of whose 2-cells is red. For the purpose of studying this case we adopt the following definitions. A $k$-dimensional simplicial complex $Q$ is called a pure $k$-complex provided that each of its simplices of dimension less than $k$ is contained in a $k$-simplex of $Q$ [see Harary (1955), p. 462]. (Such a complex is sometimes also referred to as a uniform hypergraph or $k$-graph in combinatorial literature.) The term plex will be used for a pure 2-complex. If $h$ is a graph in which each line is contained in a copy of $K_{3}$ in $H$, then there exists a plex called the plex of $H$, denoted by $P H$, whose 1-skeleton is $H$ and whose 2-cells are the copies of $K_{3}$ in $H$. Thus, for example, $P K_{n}$, the plex of the complete graph of order $n$, is the complete simplicial 2-complex on $n$ vertices. A 2-coloring of $P K_{n}$ shall mean a coloring of the 2-cells of $P K_{n}$ with two colors, green and red. For plexes $Q$ and $T, r(Q, T)$ will denote the least integer $n$ such that in every 2-coloring of $P K_{n}$, there is either a green copy of $Q$ or a red copy of $T$. In this paper we study the generalized Ramsey theory for plexes, obtaining exact values of $r(Q, T)$ for certain small plexes $Q$ and $T$. The facts established here are analogous to the graph-theoretic results obtained in Chvátal and Harary (1972), (1973), (1973a), and (1973b), and use is made of many of these results for graphs as well as of the notation of those papers.

\section{The simplest cases}

Although the methods described below could be used to determine the values of $r(Q, T)$ for a long list of pairs, and many such values have been calculated, we shall concentrate on the pairs which can be formed from the seven plexes shown in Figure 1. These seven include all of the 2-trees on fewer than six vertices in the sense of Harary and Palmer (1968), and include the smallest 
members of several classes of plexes described below, which are also explored further in another paper, Duke (1975).

For graphs $G$ and $H$ we shall use $G+n H$ to denote the join of $G$ with the graph consisting of $n$ disjoint copies of $H$.

Let $F_{n}$, the $n-f a n$, be the plex $P\left(K_{1}+P_{n+1}\right)$, that is, the plex of the graph formed by taking the join of the path $P_{n+1}$ of length $n$ with the trivial graph $K_{1}$. Continuing:

$W_{n}$, the $n$-wedge, is the plex $P\left(K_{1}+n K_{2}\right)$.

$B_{n}$, the $n$-book, is the plex $P\left(K_{2}+n K_{1}\right)$.

$D_{n}$, the $n$-disc, is the plex with vertices $v_{0}, v_{1}, \cdots, v_{n}$ whose 2 -cells are those spanned by the sets $\left\{v_{0}, v_{1}, v_{n}\right\}$ and $\left\{v_{0}, v_{i}, v_{i+1}\right\}$ for $1 \leqq i \leqq n-1$. For $n>3, D_{n}$ is the plex of the graph known as a wheel, (see Harary (1969), p. 46).
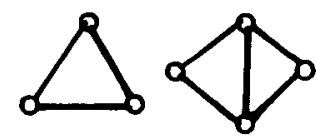

$\begin{array}{cc}P_{K_{3}} & F_{2} \\ \text { POMPLETE } & \text { FAN } \\ \text { PLEX } & \left.\text { BOOK } B_{2}\right)\end{array}$
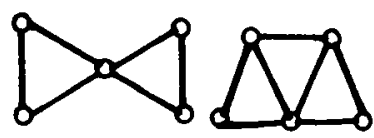

$\stackrel{W_{2}}{\text { WEDGE }}$

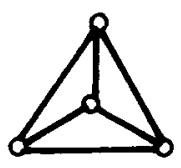

$\underset{\text { DISC }}{\stackrel{D_{3}}{2}}$

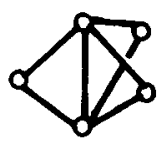

$B_{3}$
BOOK

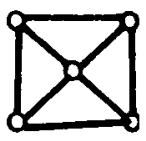

D. DISC

Fig. 1. Seven Small Plexes

The next four results are analogous to simple properties of the Ramsey numbers for graphs given in Chvátal and Harary (1962) and follow immediately from the above definitions.

$$
\begin{aligned}
& r(Q, T)=r(T, Q) \\
& Q^{\prime} \subseteq Q \text { and } T^{\prime} \subseteq T \text { imply } r\left(Q^{\prime}, T^{\prime}\right) \leqq r(Q, T) \\
& r(Q, T) \geqq \max (V(Q), V(T)), \text { where } V(A) \text { denotes the } \\
& \text { number of vertices in } A \text {. }
\end{aligned}
$$

$$
r\left(P K_{3}, Q\right)=V(Q) \text { for any pure 2-complex } Q \text {. }
$$

If $v$ is a vertex of the plex $Q$, then $G(Q-v)$ shall denote the graph whose lines are the edges of $Q$ not containing $v$. If $v$ is a vertex of each 2-cell of $Q$, we shall call $v$ an apex of $Q$. We then have the following result.

Lemma 1. If $Q$ and $T$ are plexes having apexes $a$ and $b$ respectively, then $r(Q, T) \leqq r(G(Q-a), G(T-b))+1$.

Proof. Let $v$ be a vertex of $P K_{n+1}$. Each 2-coloring of $P K_{n+1}$ induces a 
line-coloring of $G\left(P K_{n+1}-v\right)=K_{n}$ in which each line of $G\left(P K_{n+1}-v\right)$ is assigned the color of the 2-cell spanned by $v$ and that line. If $r(G(Q-a), G(T-$ $b)) \leqq n$, then there exists either a green copy of $G(Q-a)$ or a red copy of $G(T-b)$ in $G\left(P K_{n+1}-v\right)$, and hence either a green $Q$ or a red $T$ in $P K_{n+1}$.

This lemma, together with (3) and the graphical Ramsey numbers $r\left(P_{3}, P_{3}\right)=3, r\left(P_{3}, 2 K_{2}\right)=r\left(P_{3}, P_{4}\right)=r\left(P_{3}, C_{4}\right)=4$ given in Chvátal and Harary (1972b) yields the following information about plexes.

THEOREM 1. $r\left(F_{2}, F_{2}\right)=4, r\left(F_{2}, W_{2}\right)=r\left(F_{2}, F_{3}\right)=r\left(F_{2}, D_{4}\right)=5$.

The upper bound given by Lemma 1 is not always attained, even for pairs involving $F_{2}$. Since $r\left(P_{3}, C_{3}\right)=r\left(P_{3}, K_{1,3}\right)=5$ as shown in Chvátal and Harary (1972b), the bound given by Lemma 1 for both $r\left(F_{2}, D_{3}\right)$ and $r\left(F_{2}, B_{3}\right)$ is 6 . It is easy to check, however, that $r\left(F_{2}, D_{3}\right)=4$, and we consider next the value of $r\left(F_{2}, B_{3}\right)$.

THEOREM 2. $r\left(F_{2}, B_{3}\right)=5$

Proof. By (3), $r\left(F_{2}, B_{3}\right) \geqq 5$. Thus we need show only that each 2-coloring of $P K_{5}$ produces either a green $F_{2}$ or a red $B_{3}$.

Suppose $P K_{5}$ is 2-colored in such a way that there is no green copy of $F_{2}$. Let the vertices of $P K_{5}$ be $1,2,3,4$, and 5 and consider the graph $G=G\left(P K_{5}-5\right)$. The induced 2-coloring of the lines of $G$ produces no green $P_{3}$. Thus the red lines in $G$ form a copy of $K_{4}, K_{4}-x$, or $C_{4}$. If $G$ contains a red $K_{4}$ or $K_{4}-x$, then $P K_{5}$ clearly contains a red $B_{3}$. Suppose the red lines of $G$ are $(1,2),(2,3),(3,4)$, and $(1,4)$, forming a copy of $C_{4}$. Then since $P K_{5}$ contains no green $F_{2}$, the 2-cells $(1,2,3)$ and $(1,2,4)$ must be red. But then $P K_{5}$ contains a red $B_{3}$ consisting of $(1,2,3),(1,2,4)$, and $(1,2,5)$.

THEOREM 3. $r\left(W_{2}, D_{3}\right)=r\left(W_{2}, B_{3}\right)=5$.

Proof. As before, each of these values is at least 5 by (3). Let the vertices of $P K_{5}$ be $1,2,3,4$, and 5 and consider $G=G\left(P K_{5}-5\right)$. Given a 2-coloring of $P K_{5}$ which produces no green wedge $W_{2}$, the induced red subgraph of $G$ is $K_{4}$, $K_{4}-x, K_{1,3}+x, K_{1,3}$, or $K_{3}$. If $G$ contains a red $K_{4}, K_{4}-x$, or $K_{1,3}+x$, then $P K_{5}$ contains a red disc $D_{3}$ and a red book $B_{3}$, each with apex at 5 .

Suppose $G$ contains a red $K_{1,3}$ with lines $(1,2),(1,3)$, and $(1,4)$. Clearly $P K_{5}$ contains a red $B_{3}$, and since there is no green $W_{2}$, the 2 -cell $(1,2,3)$ must be red, yielding a red $D_{3},(1,2,3),(1,2,5)$, and $(1,3,5)$.

Suppose the red lines of $G$ are $(1,2),(2,4)$, and $(1,4)$, forming a $K_{3}$. Then $P K_{5}$ has a red $D_{3}$, and since $(1,2,4)$ and $(2,3,4)$ must also be red, there is a red $B_{3}$ combining these 2 -cells with $(2,4,5)$.

The reasoning of the above proof could be extended to obtain the Ramsey 
numbers for the remaining pairs which consist of $W_{2}$ and some one of the plexes shown in Figure 1, but these values will be obtained more readily by combining (2) with the next theorem.

THEOREM 4. $r\left(F_{3}, D_{3}\right)=r\left(F_{3}, D_{4}\right)=5$

Proof. By (3), $r\left(F_{3}, D_{3}\right) \geqq 5$ and $r\left(F_{3}, D_{4}\right) \geqq 5$.

Suppose that there is a 2-coloring of $P K_{5}$ which yields no green $F_{3}$. Let $v$ be a vertex of $P K_{5}$ and consider the graph $G=G\left(P K_{5}-v\right)$. The red subgraph of $G$ in the induced 2-coloring is one of $K_{4}, K_{4}-x, K_{1,3}+x, C_{4}, K_{1,3}$, or $K_{3}$. The desired result is immediate in the first two cases, while a detailed analysis of the sort used in the proofs of the two preceding theorems is required for each of the remaining cases.

The results of Theorems 3 and 4 can now be combined with (2) to show that $r\left(W_{2}, W_{2}\right)=r\left(W_{2}, F_{3}\right)=r\left(W_{2}, D_{4}\right)=r\left(F_{3}, F_{3}\right)=5$.

THEOREM 5. $r\left(F_{3}, B_{3}\right)=6$

Proof. According to Chvátal and Harary (1972b), $r\left(P_{4}, K_{1,3}\right)=5$, hence by Lemma $1, r\left(F_{3}, B_{3}\right) \leqq 6$. To see that equality holds one need only consider the 2-coloring of $P K_{5}$ in which the 2-cells $(1,2,5),(1,3,5),(1,4,5)$, and $(2,3,4)$ are colored green and the remaining 2 -cells are colored red. With this 2 -coloring there is no green $F_{3}$ and no red $B_{3}$.

THEOREM 6.

$$
r\left(D_{3}, D_{3}\right)=r\left(D_{3}, D_{4}\right)=r\left(B_{3}, D_{3}\right)=r\left(D_{4}, D_{4}\right)=r\left(D_{4}, B_{3}\right)=r\left(B_{3}, B_{3}\right)=7 \text {. }
$$

Proof. That each of these Ramsey numbers must be at least 7 follows from consideration of a specific 2-coloring of $P K_{6}$. In this 2-coloring the 2-cells of one of the colors, say red, correspond to the blocks of a balanced incomplete block design without repeated blocks and with parameters $v=6, b=10, k=3$, and $\lambda=2$, (see Hall (1967)). That is, the $b=10$ red 2-cells may be viewed as blocks (subsets) chosen from the $v=6$ vertices of $P K_{6}$ in such a way that each block contains exactly $k=3$ vertices, and each pair of distinct vertices (each edge) occurs in exactly $\lambda=2$ blocks ( 2 red 2-cells). For example, if the vertices of $P K_{6}$ are $1,2,3,4,5,6$, then the 2 -cells $(1,2,4),(1,2,6),(1,3,4),(1,3,5),(1,5,6)$, $(2,3,5),(2,3,6),(2,4,5),(3,4,6)$, and $(4,5,6)$ form such a triple system and may be taken to be the red 2-cells. It follows that with this 2-coloring, for any vertex $v$ of $P K_{6}$, the red 2-cells containing $v$ and the green 2-cells containing $v$ each form a monochromatic $D_{5}$ with apex at $v$. There exists no monochromatic $D_{3}$ or $D_{4}$ in $P K_{6}$. Since $\lambda=2$, while each edge of $P K_{6}$ is contained in a total of four 2-cells, there exists no monochromatic $B_{3}$. Hence each of the Ramsey numbers listed in the theorem must be at least 7 . 
That $r\left(D_{3}, D_{3}\right)=r\left(D_{4}, D_{4}\right)=r\left(D_{4}, B_{3}\right)=r\left(B_{3}, B_{3}\right)=7$ follows now from Lemma 1 and the graphical Ramsey numbers

$$
r\left(K_{3}, K_{3}\right)=r\left(C_{4}, C_{4}\right)=r\left(C_{4}, K_{1,3}\right)=r\left(K_{1,3}, K_{1,3}\right)=6
$$

listed in Chvátal and Harary (1972b).

It remains to show that $r\left(B_{3}, D_{3}\right) \leqq 7$ and $r\left(D_{3}, D_{4}\right) \leqq 7$.

For the first of these, suppose there is a 2-coloring of $P K$, yielding no green $B_{3}$ and no red $D_{3}$. Then each edge of $P K_{7}$ is adjacent to at least 3 red 2-cells. There are 21 edges in $P k_{7}$ and each 2-cell is adjacent to 3 edges, so it follows that there must be at least 21 red 2-cells. On the other hand, since there exists no red $D_{3}$, each copy of $P K_{4}$ in $P K_{7}$ must include at least 2 green 2-cells. But since each 2-cell is in 4 of the 35 copies of $P K_{4}$ in $P K_{7}$, there must be at least 18 green 2-cells. These conclusions contradict the fact that $P K$, has only 352 -cells in all.

Now suppose $P K$, is 2 -colored in such a way that there exists no green copy of $D_{3}$ and no red copy of $D_{4}$. As above there must be at least 182 -cells colored red. It follows that some vertex is adjacent to at least 8 red 2-cells. Let the vertices of $P K_{7}$ be $1,2, \cdots, 7$, with 7 incident with at least eight red 2-cells. Then the graph $G=G\left(P K_{7}-7\right)$ has at least eight red lines in the induced 2-coloring. Some point of $G$ is adjacent to at least three red lines. We may assume that $(1,2),(1,4)$, and $(1,6)$ are red lines of $G$. Since $G$ contains no red $C_{4}$, at least two of the lines $(2,3),(3,4)$, and $(3,6)$ must be green. We may suppose $(2,3)$ and $(3,4)$ are green. Since $G$ has no green $C_{3},(2,4)$ must then be red. Now one of the lines $(2,5)$ and $(4,5)$, say $(4,5)$, is green. Since $(3,4)$ and $(4,5)$ are green, $(3,5)$ must be red. Since $(1,2),(1,6)$, and $(2,4)$ are red, $(4,6)$ must be green. Since $(3,4),(4,5)$, and $(4,6)$ are green, $(3,6)$ and $(5,6)$ must be red, and hence $(2,5)$ and $(1,3)$ must be green. Now one of $(1,5)$ and $(2,6)$ must be green, since $(1,2)$ and $(5,6)$ are red. By the symmetry of the colors indicated thus far, we may assume that $(1,5)$ is green. Thus the 2-cells $(1,3,7)(2,3,7),(3,4,7),(1,5,7),(4,5,7)$ and $(2,5,7)$ are green in $P K_{7}$. Since there is no green $D_{3},(1,2,3),(1,3,4),(1,4,5)$, and $(1,2,5)$ must be red. But these 2-cells then form a red $D_{4}$. This contradiction shows that $r\left(D_{3}, D_{4}\right) \leqq 7$, and hence concludes the proof of the theorem.

The above results taken together give the Ramsey numbers for all of the 28 pairs that can be drawn from the list of plexes shown in Figure 1 and this information is summarized in the table.

\section{Some pairs involving $P K_{4}$}

By (4), $r\left(P K_{3}, P K_{4}\right)=4$. It is clear that $r\left(F_{2}, P K_{4}\right)>4$. The fact that any 2-coloring of $P K_{5}$ with more than two green 2-cells contains a green $F_{2}$ leads immediately to $r\left(F_{2}, P K_{4}\right)=5$. The next theorem gives several other values for such pairs. 
TABLE 1

Ramsey numbers for some small plexes

\begin{tabular}{cccccccc}
\hline & $P_{K_{3}}$ & $F_{2}$ & $W_{2}$ & $F_{3}$ & $D_{3}$ & $B_{3}$ & $D_{4}$ \\
\hline$P K_{3}$ & 3 & 4 & 5 & 5 & 4 & 5 & 5 \\
$F_{2}$ & & 4 & 5 & 5 & 4 & 5 & 5 \\
$W_{2}$ & & & 5 & 5 & 5 & 5 & 5 \\
$F_{3}$ & & & & 5 & 5 & 6 & 5 \\
$D_{3}$ & & & & 7 & 7 & 7 \\
$B_{3}$ & & & & & 7 & 7 \\
$D_{4}$ & & & & & & 7 \\
\hline
\end{tabular}

THEOREM 7.
(i) $r\left(W_{2}, P K_{4}\right)=6$;
(ii) $r\left(F_{3}, P K_{4}\right)=7$
(iii) $r\left(B_{3}, P K_{4}\right)=8$;
(iv) $r\left(D_{3}, P K_{4}\right)=8$

Proof of (i). A 2-coloring of $P K_{5}$ in which only the 2-cells of a single $P K_{4}$ are colored green has no green $W_{2}$ and no red $P K_{4}$. Thus $r\left(W_{2}, P K_{4}\right) \geqq 6$.

Suppose $P K_{6}$ is 2 -colored in such a way that there exists no green $W_{2}$. Let the vertices of $P K_{6}$ be $1,2, \cdots, 6$, and consider the graph $G=G\left(P K_{6}-6\right)$. Since $r\left(2 K_{2}, K_{4}-x\right)=5$, the induced 2-coloring of $G$ yields a red $K_{4}-x$. Suppose the lines $(1,2),(2,3),(1,3),(3,5)$ and $(1,5)$ form such a red $K_{4}-x$. Then if either of the 2-cells $(1,2,3)$ or $(1,3,5)$ is red, $P K_{6}$ contains a red $P K_{4}$ spanned by this 2 -cell and the vertex 6 . Suppose then that $(1,2,3)$ and $(1,3,5)$ are green. Since $(1,3,5)$ forms a copy of $W_{2}$ with $(3,4,6)$ and with $(4,5,6)$, both of these must be red. Likewise, since $(1,2,3)$ is green, $(3,4,5)$ must be red. Thus each 2 -cell of the $P K_{4}$ with vertices $3,4,5$, and 6 is red.

Proof of (ii). The 2-coloring of $P K_{6}$ in which the only green 2-cells are $(1,2,4),(1,2,6),(1,3,5),(2,3,5),(3,4,6)$, and $(4,5,6)$ has no green $F_{3}$ and no red $P K_{4}$. Thus $r\left(F_{3}, P K_{4}\right) \geqq 7$.

Suppose there is a 2-coloring of $P K_{7}$ in which there is no green $F_{3}$ and no red $P K_{4}$. Each of the 35 copies of $P K_{4}$ has at least one green 2-cell, and each green 2 -cell is in four of these copies. Hence there must be at least nine green 2-cells, and some $\mathrm{PK}_{4}$ must contain at least two green 2 -cells. Suppose $(1,2,3)$ and $(1,2,4)$ are both green. These two 2 -cells together with any one of $(1,4,5)$, $(1,4,6)$, or $(1,4,7)$ would form a $F_{3}$ with apex at 4 . We may suppose then that $(1,4,5),(1,4,6)$, and $(1,4,7)$ are red. Similarly, the 2 -cells $(2,4,5),(2,4,6)$, $(2,4,7),(1,3,5),(1,3,6),(1,3,7),(2,3,5),(2,3,6)$, and $(2,3,7)$ must all be red. 
Each $P K_{4}$ having one vertex in the set $\{1,2\}$, one in $\{3,4\}$, and two vertices in $\{5,6,7\}$ must contain a green 2 -cell, so either both $(1,5,6)$ and $(2,5,6)$ are green or both $(3,5,6)$ and $(4,5,6)$ are green. Likewise $(1,5,7)$ and $(2,5,7)$ are both green or $(3,5,7)$ and $(4,5,7)$ are both green, and $(1,6,7)$ and $(2,6,7)$ are both green or $(3,6,7)$ and $(4,6,7)$ are both green. Thus the green 2-cells include at least two of the pairs $\{(1,5,6),(2,5,6)\},\{(1,5,7),(2,5,7)\}$, and $\{(1,6,7),(2,6,7)\}$ or two of the pairs $\{(3,5,6),(4,5,6)\},\{(3,5,7),(4,5,7)\}$, and $\{(3,6,7),(4,6,7)\}$. In either case we have a green $F_{3}$.

Proof of (iii). To show that $r\left(B_{3}, P K_{4}\right) \geqq 8$ we need a 2-coloring of $P K_{7}$ which produces no green $B_{3}$ and no red $P K_{4}$. Such a 2-coloring can be obtained by letting the 2-cells colored green be those corresponding to the blocks or triples of two disjoint Setiner triple systems on $v=7$ vertices, each with $b=7$ distinet blocks. An example of such a pair of triple systems is $\{(1,2,4),(1,3,5)$, $(1,6,7),(2,3,6),(2,5,7),(3,4,7),(4,5,6)\}$ and $\{(1,2,6),(1,3,4),(1,5,7),(2,3,7)$, $(2,4,5),(3,5,6),(4,6,7)\}$.

Each pair of vertices (each edge) is then contained in exactly $\lambda=1$ block in each triple system, and each vertex is in 3 blocks in each system. For each vertex $u$ of $P K_{7}$ with this 2 -coloring, the green 2-cells containing $u$ form a copy of $D_{6}$ with apex at $u$, three of whose 2-cells are in one of the triple systems and three in the other. Thus $P K_{7}$ contains no green $B_{3}$ (or $D_{3}, D_{4}$, or $D_{5}$ ). Suppose that there exists a $P K_{4}$, say $(1,2,3,4)$, having no green 2-cell. Each edge of this $P K_{4}$ is on exactly two green 2 -cells, each with its third vertex in the set $\{5,6,7\}$. But the edges joining the vertices of $(1,2,3,4)$ to the other vertices are also each on exactly two green 2-cells, so each of 5,6 , and 7 must be on four green 2-cells whose other vertices are in $(1,2,3,4)$. This leads to three green copies of $D_{4}$ with apexes at 5, 6, and 7 and all other vertices in $(1,2,3,4)$. But as indicated, $P K_{7}$ contains no green $\mathrm{D}_{4}$ with such a 2 -coloring.

Now suppose that there exists a 2-coloring of $P K_{8}$ in which there is no green $B_{3}$ and no red $P K_{4}$. Each copy of $P K_{4}$ contains at least one green 2-cell. Since there are 70 such copies of $P K_{4}$ and each 2-cell is in exactly five of these, there are at least 14 green 2-cells. Some vertex must be in at least six green 2-cells. Suppose 8 is such a vertex and consider the graph $G=G\left(P K_{8}-8\right)$. This graph has at least six green lines in the induced 2-coloring, while each point is adjacent to at most two green lines since there is no green $B_{3}$ in $P K_{8}$. Hence the partition (or degree sequence) of the green subgraph, $H$, of $G$ must be $(2,2,2,2,2,2,2)$, $(2,2,2,2,2,1,1)$, or $(2,2,2,2,2,2,0)$ (see Harary (1969), p. 57). If the partition is $(2,2,2,2,2,2,2), H$ is either (a) the cycle $C_{7}$ or (b) the disjoint union of $K_{3}$ and $C_{4}$. If the partition is $(2,2,2,2,2,1,1), H$ is (c) the path $P_{7}$, (d) the disjoint union of $K_{2}$ and $C_{5}$, (e) the disjoint union of $P_{3}$ and $C_{4}$, or (f) the disjoint union of $P_{4}$ 
and $C_{3}$. If the partition is $(2,2,2,2,2,2,0), H$ is either $(\mathrm{g})$ the disjoint union of two copies of $K_{3}$ plus an isolated point, or (h) $C_{6}$ plus an isolated point.

Suppose there were red lines in $G$ forming three copies of $K_{3}$ all with a single line in common. Each of these copies of $K_{3}$ would correspond to a red $D_{3}$ in $P K_{8}$. The fourth 2-cell in the $P K_{4}$ which contains any one of these $D_{3}$ would have to be green. This would produce three green 2-cells sharing a common edge, the line common to the three copies of $K_{3}$ in $G$. but this produces a green $B_{3}$ which was assumed not to exist. Thus no such configuration of red lines is possible in $G$. It follows that the green subgraph of $G$ can not be of types (b), (c), (e), (f), or (g).

If there are red lines in $G$ which form two line-disjoint copies of $K_{4}-x$ having only one or both of their points of degree two in common, then there are four red copies of $D_{3}$ in $P K_{8}$. Again the fourth 2-cell of the $P K_{4}$ that contains one of these copies of $D_{3}$ must be green. The lines joining the two points of degree three in each $K_{4}-x$ can be on no more than two green 2 -cells in $P K_{8}$. Hence all of the 2-cells in the copy of $P K_{4}$ which has a vertices the four points of degree three in the two copies of $K_{4}-x$ must be red. But there is no red $P K_{4}$, so no such configuration of red lines exists in $G$. The green subgraph of $G$ can not be of the remaining types (a), (d), or (h). This contradiction completes the proof of (iii).

Proof of (iv). Consideration of the 2-coloring of $P K_{7}$ described in the proof of (iii) shows that $r\left(D_{3}, P K_{4}\right) \geqq 8$.

An argument similar to those given for (i), and (ii), but considerably longer, may be used to complete the proof in this case. One begins by showing, with the aid of (iii), that if there exists a 2-coloring of $P K_{8}$ which yields no green $D_{3}$ and no red $\mathrm{PK}_{4}$, then in this 2-coloring some edge must be incident with exactly three green 2-cells. It follows next that a certain nine of the 2 -cells which share edges with these green 2-cells must be red. Continuing to determine in turn the colors required for a large number of the other 2-cells, one is able to show in the end that no such 2-coloring exists.

The Ramsey numbers just considered are given in the next table which, together with Table 1 above, contains all of our exact results.

TABLE 2

Ramsey numbers of the complete plex $P K_{4}$

\begin{tabular}{ccccccc} 
& $P K_{3}$ & $F_{2}$ & $W_{2}$ & $F_{3}$ & $D_{3}$ & $B_{3}$ \\
\hline$P K_{4}$ & 4 & 5 & 6 & 7 & 8 & 8
\end{tabular}


The existence of the 2-coloring of $P K_{7}$ described in the proof of part (iii) of Theorem 7 shows that $r\left(D_{4}, P K_{4}\right) \geqq 8$. It seems likely that $r\left(D_{4}, P K_{4}\right)$ is at least 9 , although this has not been shown. It may be that some of the results or techniques of Theorem 6 will be useful in determining the value of $r\left(P K_{4}, P K_{4}\right)$, or at least in improving the known bounds for this Ramsey number, but at present the best bounds still seem to be those of Sobczyk (1967) who showed that $14 \leqq r\left(P K_{4}, P K_{4}\right) \leqq 18$.

The techniques introduced here are used in Duke (1975) to derive formulae giving the Ramsey number for several of the families of pairs which can be constructed using plexes from among the $W_{n}, F_{n}, B_{n}, D_{n}$, and $P K_{n}$. The Ramsey numbers for the smallest pairs in these families occur in the tables above, and in several cases these first values are exceptions to the general formulae.

In the proofs of Theorems 6 and 7 use was made of examples from the study of block designs. In Duke (1975) further attention is devoted to the close relationship between certain formulae for Ramsey numbers of pairs of plexes and various results and open questions involving Steiner triple systems and other designs.

\section{References}

S. A. Burr (1974), 'Generalized Ramsey theory for graphs - a survey', Graphs and Combinatorics (R. Bari and F. Harary, eds.). (Springer-Verlag, Berlin, 52-75).

V. Chvátal, F. Harary (1972), 'Generalized Ramsey theory for graphs', Bull. Amer. Math. Soc. 78, $423-426$.

V. Chvátal, F. Harary (1972a), 'Generalized Ramsey theory for graphs II, Small diagonal numbers', Proc. Amer. Math Soc. 32, 389-394.

V. Chvátal, F. Harary (1972b), 'Generalized Ramsey theory for graphs III, Small off-diagonal numbers', Pacific J. Math. 41, 335-345.

V. Chvátal, F. Harary (1973), 'Generalized Ramsey theory for graphs I, Diagonal numbers', Periodica Math. Hungar. 3, 113-122.

R. A. Duke (1975), 'Ramsey numbers of families of 2-complexes', (to appear).

J. E. Graver and J. Yackel (1968), 'Some graph theoretic results associated with Ramsey's theorem', J. Combinatorial Theory 4, 125-175.

M. Hall, Jr. (1967), Combinatorial Theory (Blaisdell, 1967).

F. Harary (1955), 'The number of linear, directed, rooted, and connected graphs', Trans. Amer. Math. Soc. 78, 445-463.

F. Harary (1969), Graph Theory (Addison-Wesley, 1969).

F. Harary (1974), 'A survey of generalized Ramsey theory', Graphs and Combinatorics (R. Bari and F. Harary, eds.). (Springer-Verlag, Berlin, 1974), 10-17.

F. Harary, P. Hell (1974), 'Generalized Ramsey theory for graphs V, The Ramsey number of a digraph', Bull. London Math.'Soc. 6, 175-182.

F. Harary, E. M. Palmer (1968), 'On acyclic simplicial complexes', Mathematika 15, $115-122$.

F. Harary, G. Prins (1974), 'Generalized Ramsey theory for graphs IV, The Ramsey multiplicity of a graph', Networks 4, 163-173. 
F. P. Ramsey (1930), 'On a problem of formal logic', Proc. London Math. Soc. 30, 264-286.

H. J. Ryser (1963), Combinatorial Mathematics. (Mathematical Association of America, 1963).

A. Sobczyk (1967), 'Multi-functions associated with Steiner systems', Notices Amer. Math. Soc. 14, 9-25.

School of Mathematics,

Georgia Institute of Technology,

Atlanta, Georgia 30332,

U.S.A. 